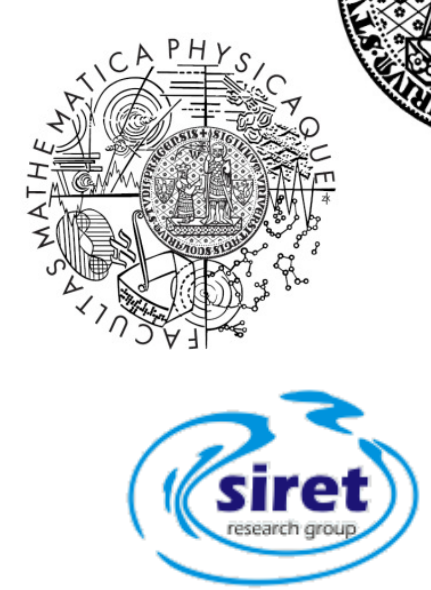

http://prisma.dcc.uchile.cl

\section{PRISMA}

Pattern recognition, indexing, and

similarity search in multimedia archives

\title{
Non-Metric Similarity Search Problems in Very Large Collections
}

Benjamin Bustos, University of Chile Tomáš Skopal, Charles University in Prague 


\section{Outline of the tutorial}

- Benjamin

- Introduction

- The non-metric case of similarity

- Case study 1 -image retrieval

- Case study 2 - time series retrieval

- Tomáš

- Case study 3 - protein retrieval

- Indexing non-metric spaces

- Challenges 


\section{Introduction}

- Similarity search

> Search for "similar objects" (subjective)

- Content-based similarity search: query by example:

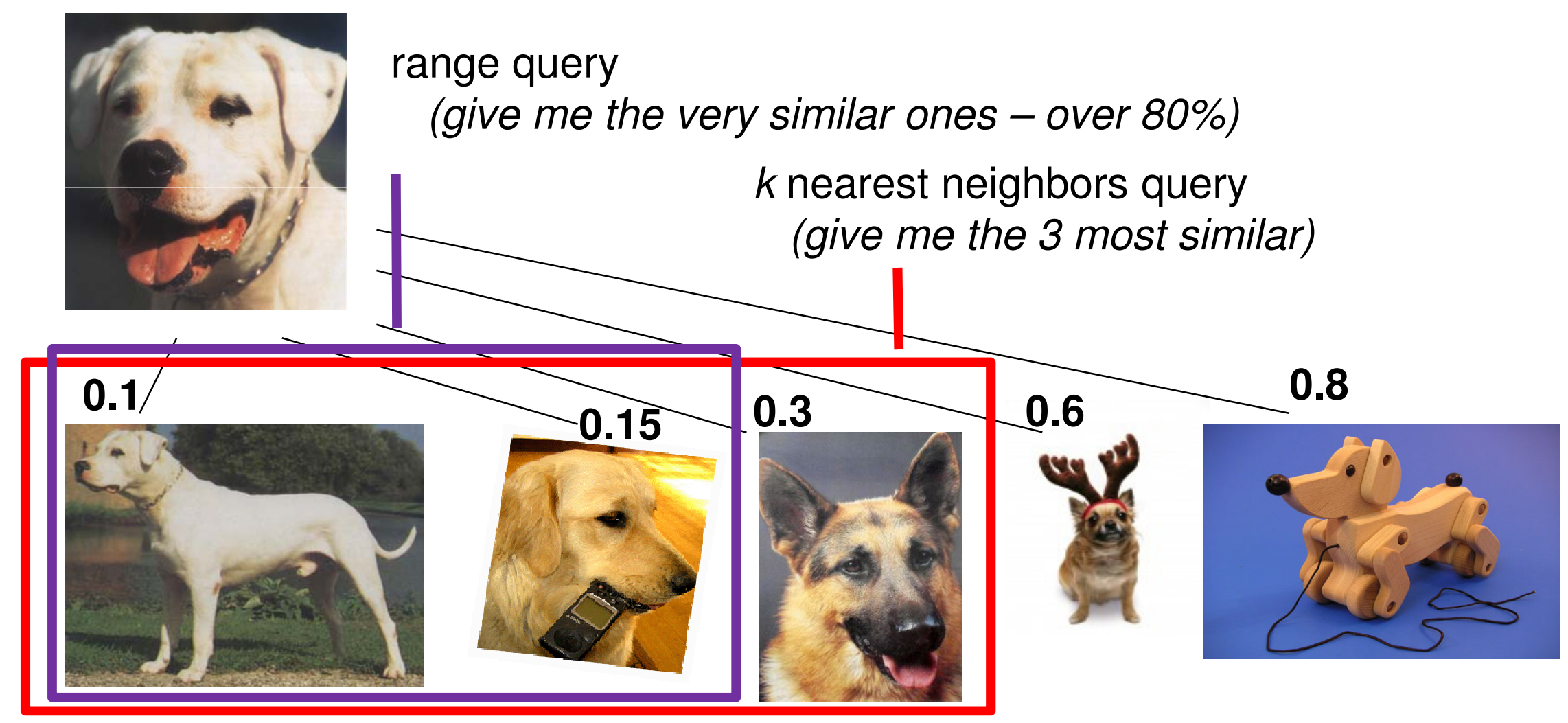

ICDE 2011, Hannover, Germany 


\section{Introduction}

- Application examples of similarity search

- Multimedia retrieval

- Scientific databases

- Biometry

- Pattern recognition

- Manufacturing industry

- Cultural heritage

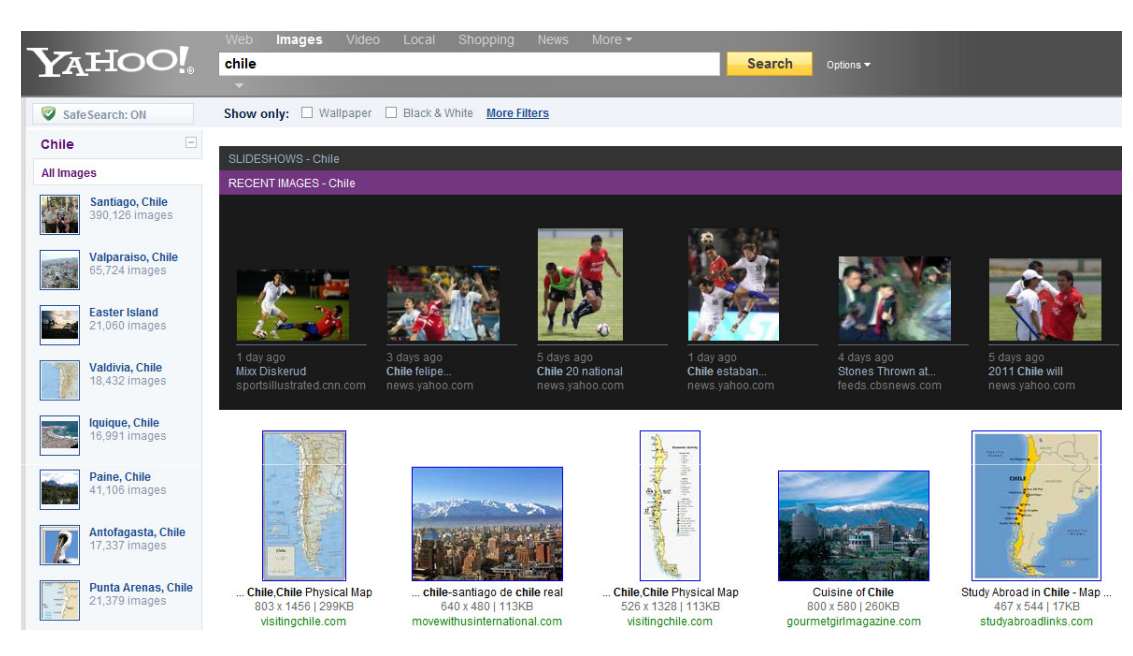

- Etc.
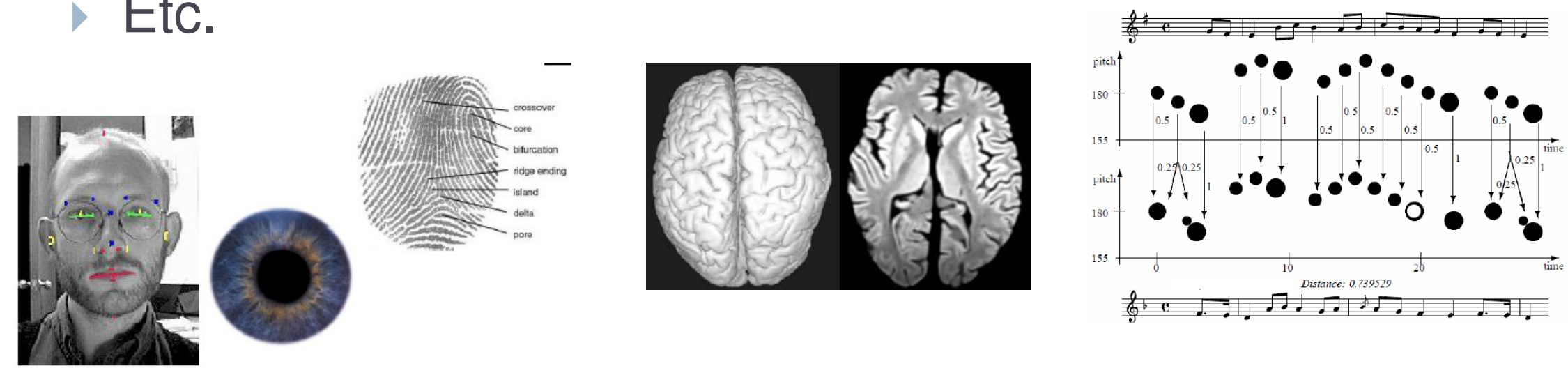

ICDE 2011, Hannover, Germany 


\section{Introduction}

- Metric similarity

- Dissimilarity function $\delta$ (the distance), universe $\mathbf{U}$, database $\mathbf{S} \subset \mathbf{U}$, objects $\mathrm{x}, \mathrm{y}, \mathrm{z} \in \mathbf{U}$

- The higher $\delta(x, y)$, the more dissimilar objects $x, y$ are

- Topological properties

$$
\begin{aligned}
\delta(x, y) & =0 \Leftrightarrow x=y & \text { identity } \\
\delta(x, y) & \geq 0 & \text { non-negativity } \\
\delta(x, y) & =\delta(y, x) & \text { symmetry } \\
\delta(x, y)+\delta(y, z) & \geq \delta(x, z) & \text { triangle inequality }
\end{aligned}
$$

- Pros of metric approach

- Well-studied in mathematics (many known metrics)

- Postulates support common assumptions on similarity

- Allows efficient indexing and search (metric indexing) 


\section{Introduction}

- Cons of metric approach:

> It may not correctly model the "human" notion of similarity

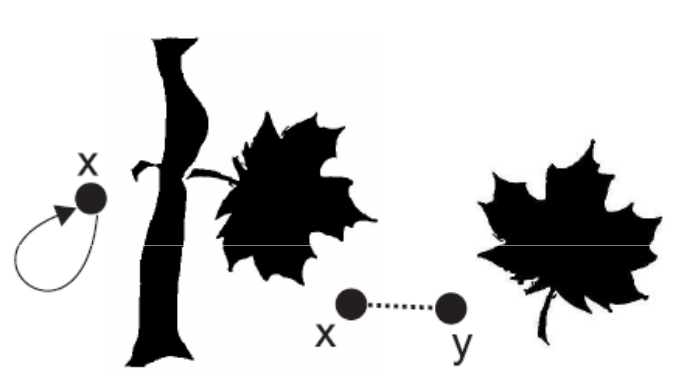

(a) $\delta(x, x)>0$

(b) $\delta(x, y)=0$

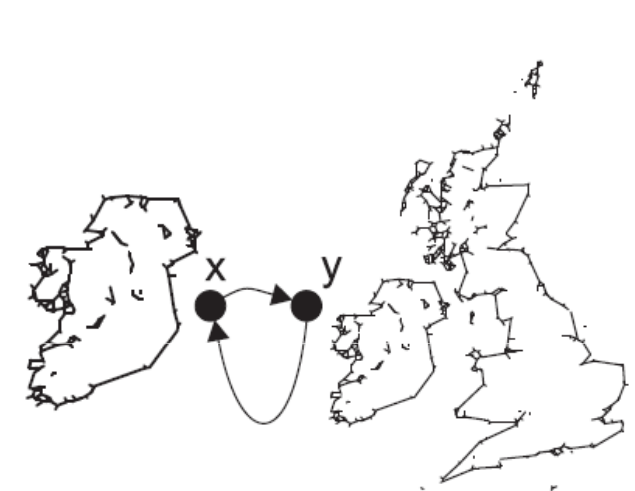

(c) $\delta(\mathrm{x}, \mathrm{y})<\delta(\mathrm{y}, \mathrm{x})$

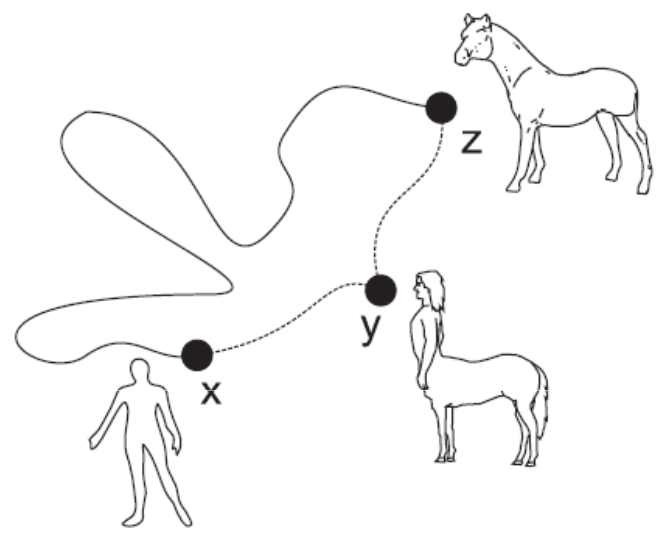

(d) $\delta(\mathrm{x}, \mathrm{y})+\delta(\mathrm{y}, \mathrm{x})<\delta(\mathrm{x}, \mathrm{z})$

> Reflexivity and non-negativity:

$\square$ single object could be viewed as self-dissimilar

$\square$ two distinct object could be viewed as identical

- Symmetry - comparison direction could be important

- Triangle inequality - similarity is not transitive 


\section{The non-metric case of similarity}

-What is non-metric?

- Generally: a distance function that does not satisfy some (or all) properties of a metric

- This could include:

- Context-dependent similarity functions

- Dynamic similarity functions

- For this tutorial: similarity functions that are "contextfree and static"

- Similarity between two objects is constant whatever the context is, i.e., regardless of time, user, query, other objects in database, etc. 


\section{The non-metric case of similarity}

- Motivation

- Robustness

- A robust function is resistant to outliers (noise or deformed objects), that would otherwise distort the similarity distribution within a given set of objects

- Having objects $x$ and $y$ and a robust function $\delta$, an extreme change in a small part of $x$ 's descriptor should not imply an extreme change of $\delta(x, y)$.
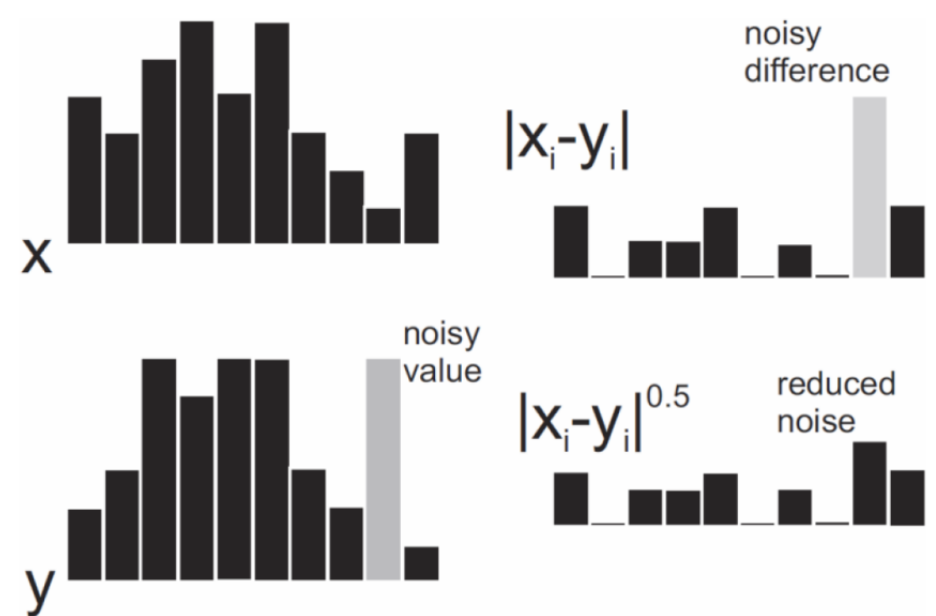


\section{The non-metric case of similarity}

- Motivation

- Locality

A locally sensitive function is able to ignore some portions of the compared objects

- The locality is usually usec to privilege similarity befor dissimilarity, hence, we rather search for similar parts in two objects than for dissimilar parts

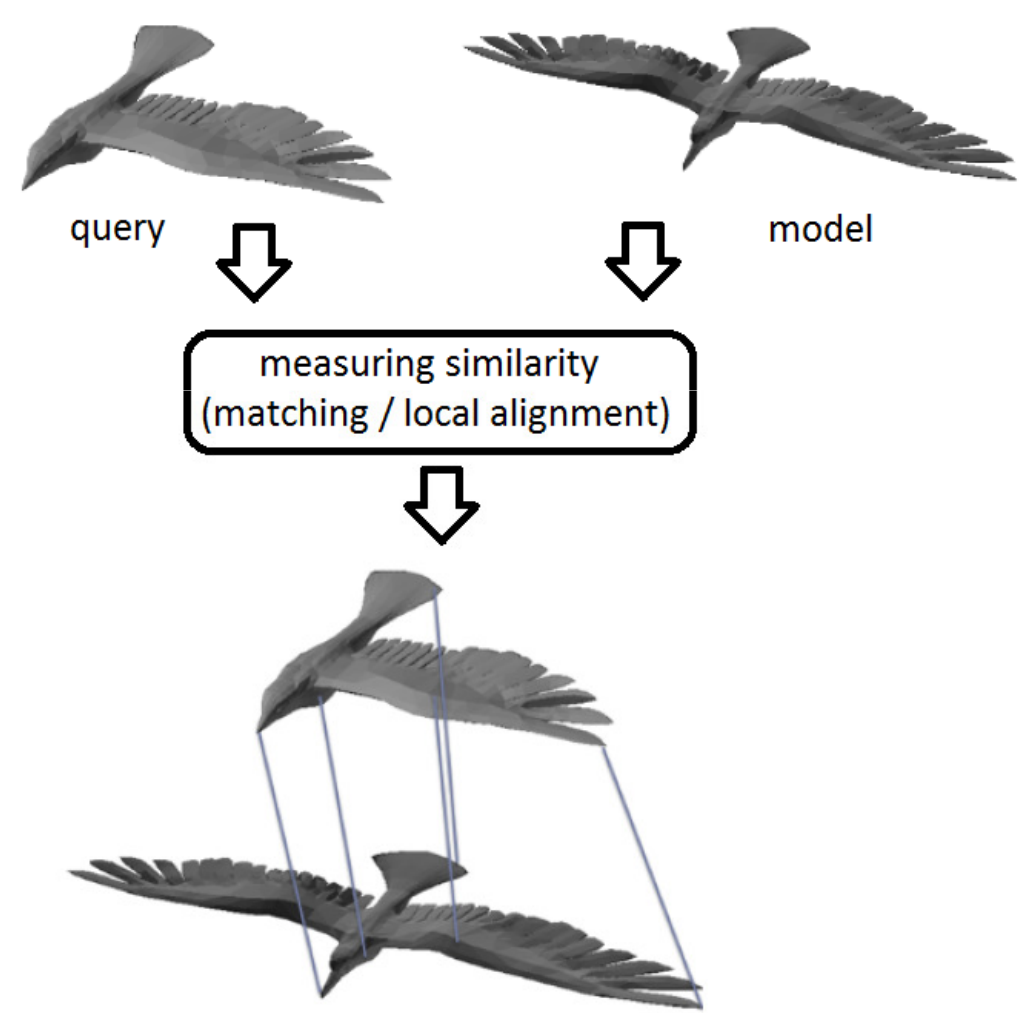




\section{The non-metric case of similarity}

- Motivation

- Comfort/freedom of modeling

- The task of similarity search should serve just as a computer based tool in various professions

- Domain experts should not be bothered by some "artificial" constraints (metric postulates)

$\square$ Enforcement of metric may represent an unpleasant obstacle

- Freedom of modeling

$\square$ Complex heuristic algorithms

$\square$ Black-box similarity

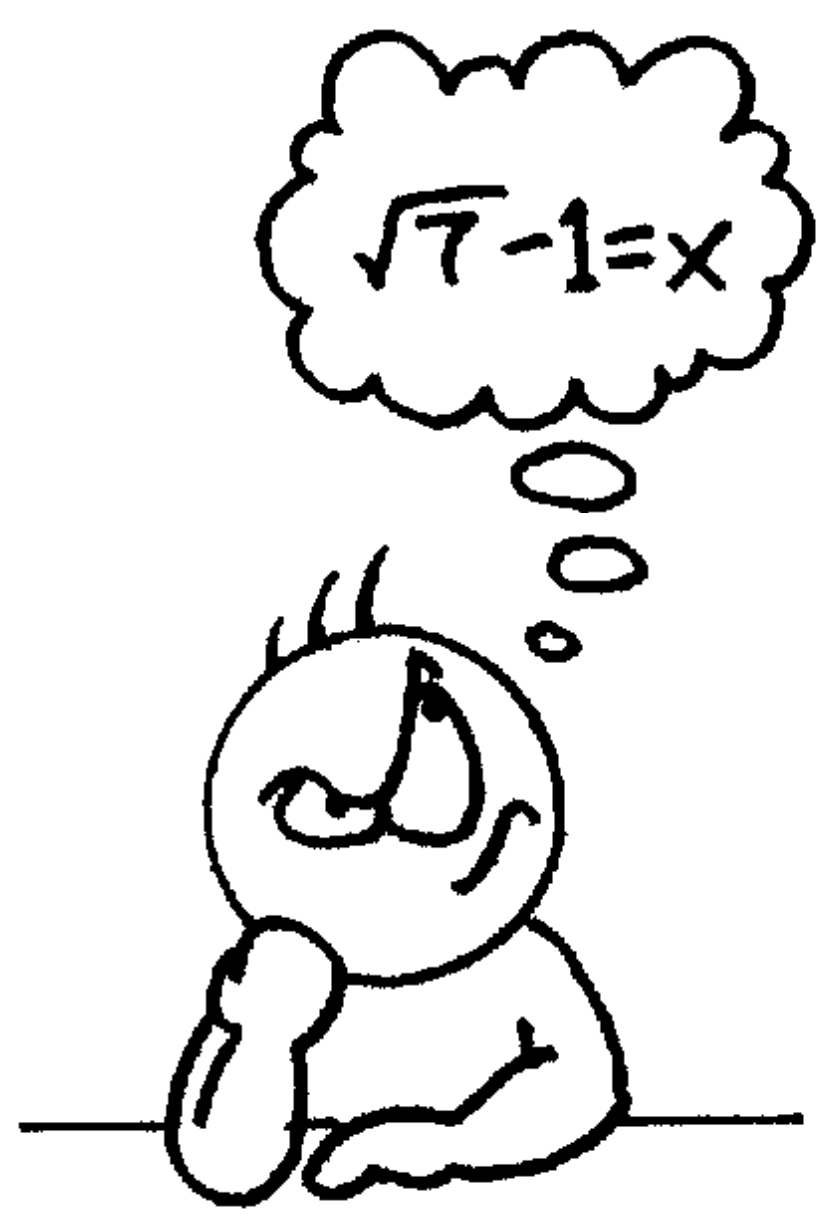




\section{The non-metric case of similarity}

\section{- Examples of general non-metric functions}

- Fractional $L p$ distances $(p<1)$, Sequence alignment distance

$L_{p}(x, y)=\left(\sum_{i=1}^{d}\left|x_{i}-y_{i}\right|^{p}\right)^{1 / p} \delta_{S A D}(x, y, i, j)=\min \left\{\begin{array}{c}c\left(x_{i}, y_{j}\right)+\delta_{S A D}(x, y, i+1, j+1) \\ c\left(-y_{j}\right)+\delta_{S A D}(x, y, i, j+1) \\ c\left(x_{i},-\right)+\delta_{S A D}(x, y, i+1, j)\end{array}\right.$

- Cosine similarity

$$
s_{\cos }(x, y)=\frac{\sum_{i=1}^{d} x_{i} y_{i}}{\sqrt{\sum_{i=1}^{d} x_{i}{ }^{2} \cdot \sum_{i=1}^{d} y_{i}{ }^{2}}}
$$

- Earth Mover's distance

$$
\delta_{E M D}(x, y)=\min \left\{\sum_{i=1}^{d} \sum_{j=1}^{d} c_{i j} f_{i j}\right\}
$$
subject to

$$
\begin{aligned}
f_{i j} & \geq 0 \\
\sum_{i=1}^{d} f_{i j} & =y_{j} \forall j=1, \ldots, d \\
\sum_{j=1}^{d} f_{i j} & =x_{i} \forall i=1, \ldots, d
\end{aligned}
$$




\section{Case study 1 - image retrieval}

- The problem: find similar images to a given one
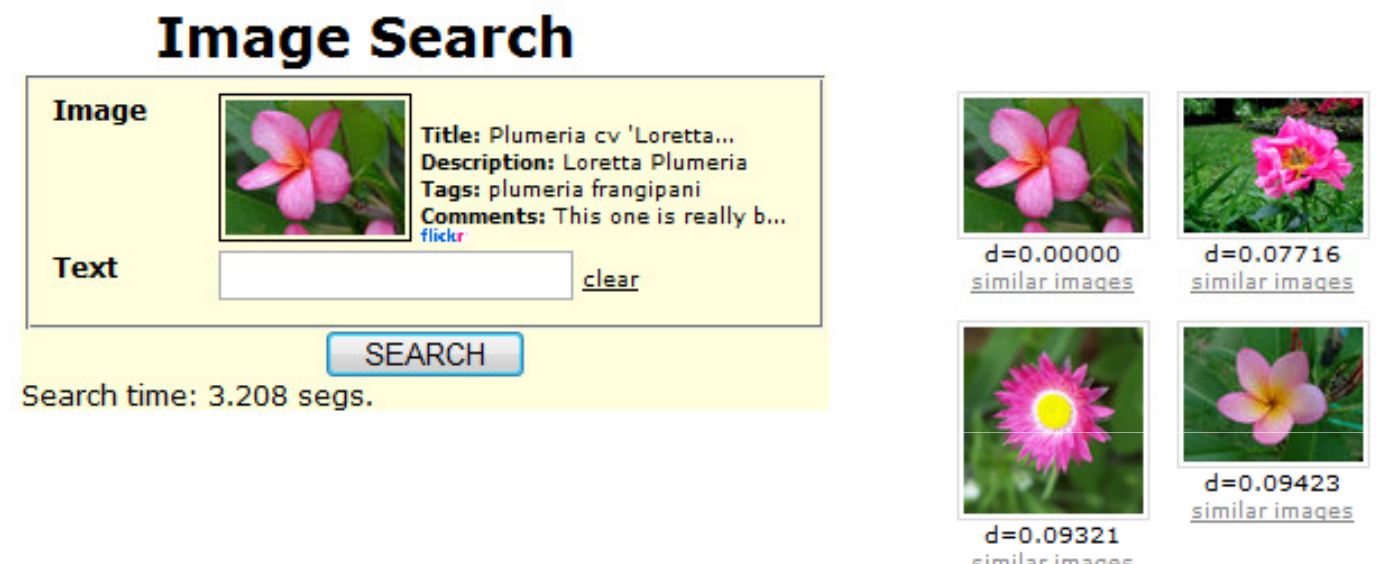

$d=0.07716$

similar images
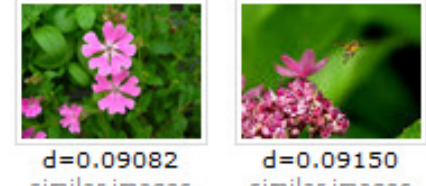

$d=0.09150$

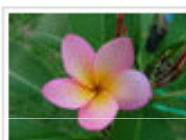

$d=0.09423$

similar images

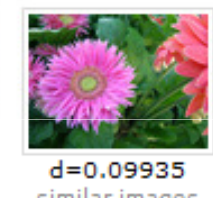

similar images

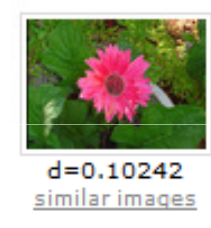

- Query specification: Text (metadata), Content-based, Sketch-based, combination

PRISMA Image Search:

http://prisma.dcc.uchile.cl/ImageSearch/
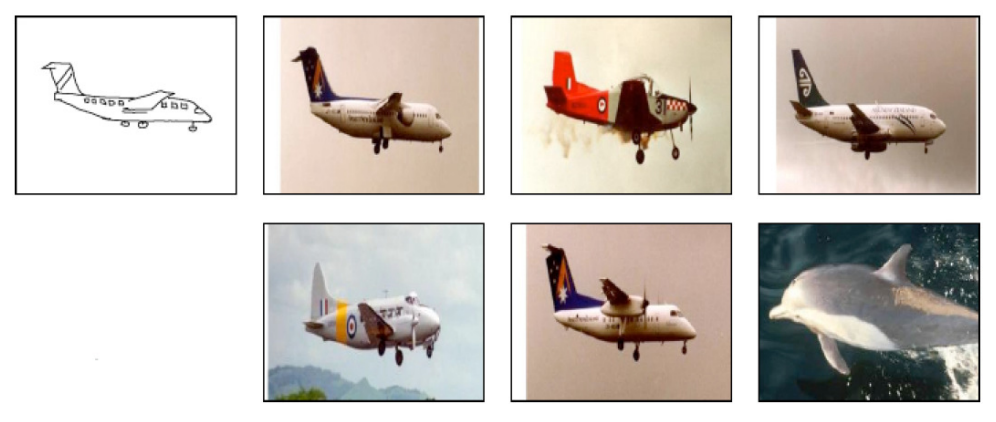


\section{Case study 1 - image retrieval}

- Image descriptors

> High-level features: concepts

- Metadata

$\square$ Title, tags, etc.

- Click information

$\square$ Web-logs

$\square$ Also carries semantic information

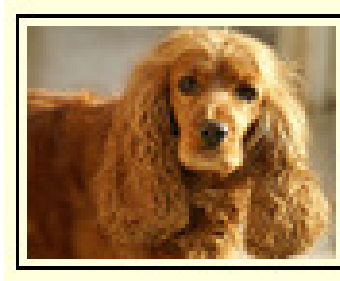

Title: She is a Lady

Description: Prissy, sun-lit.

Tags: coker spaniel coker...

Comments: Prissy is beautiful....

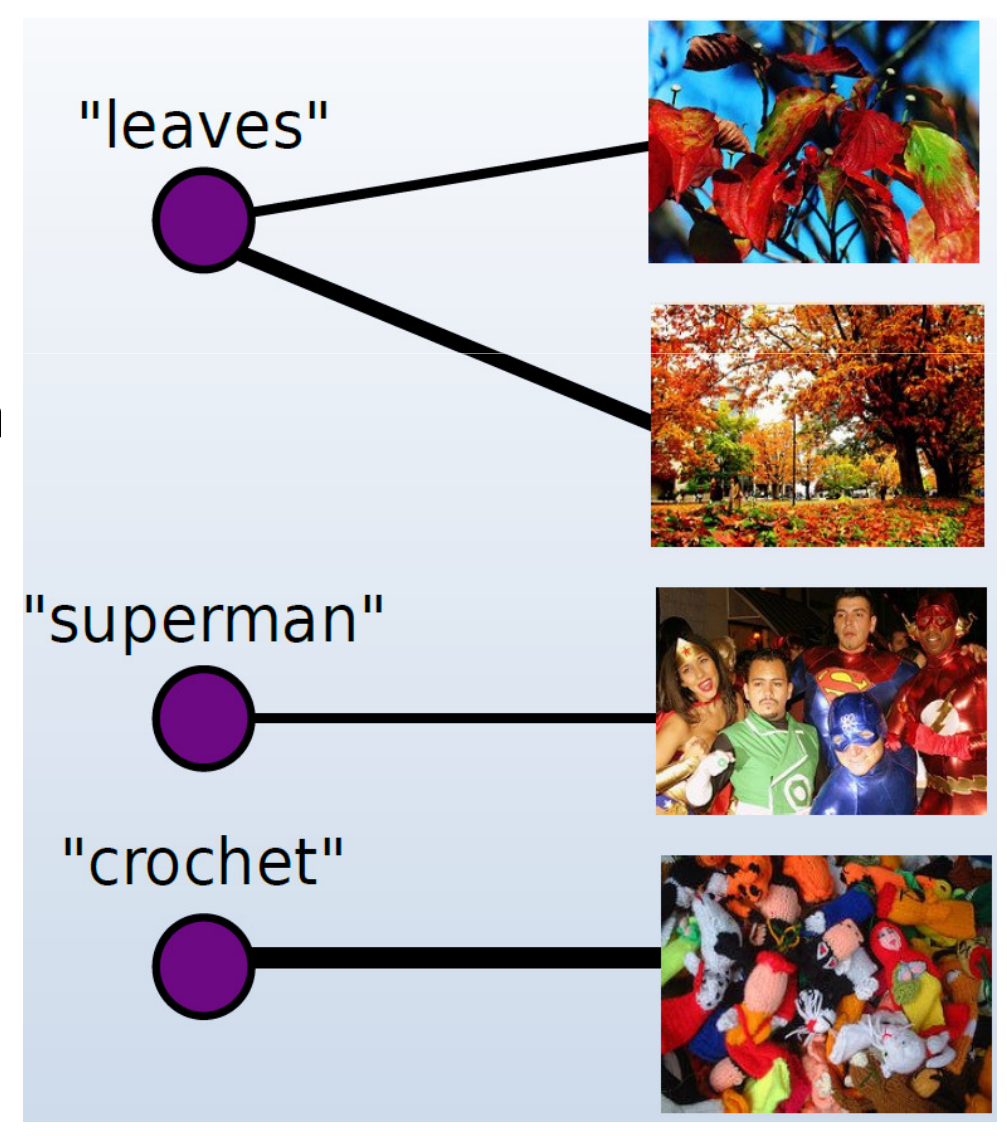




\section{Case study 1 - image retrieval}

- Image descriptors

- Low-level features: visual attributes

- Color, texture, shape, edges

- Global vs. local descriptors
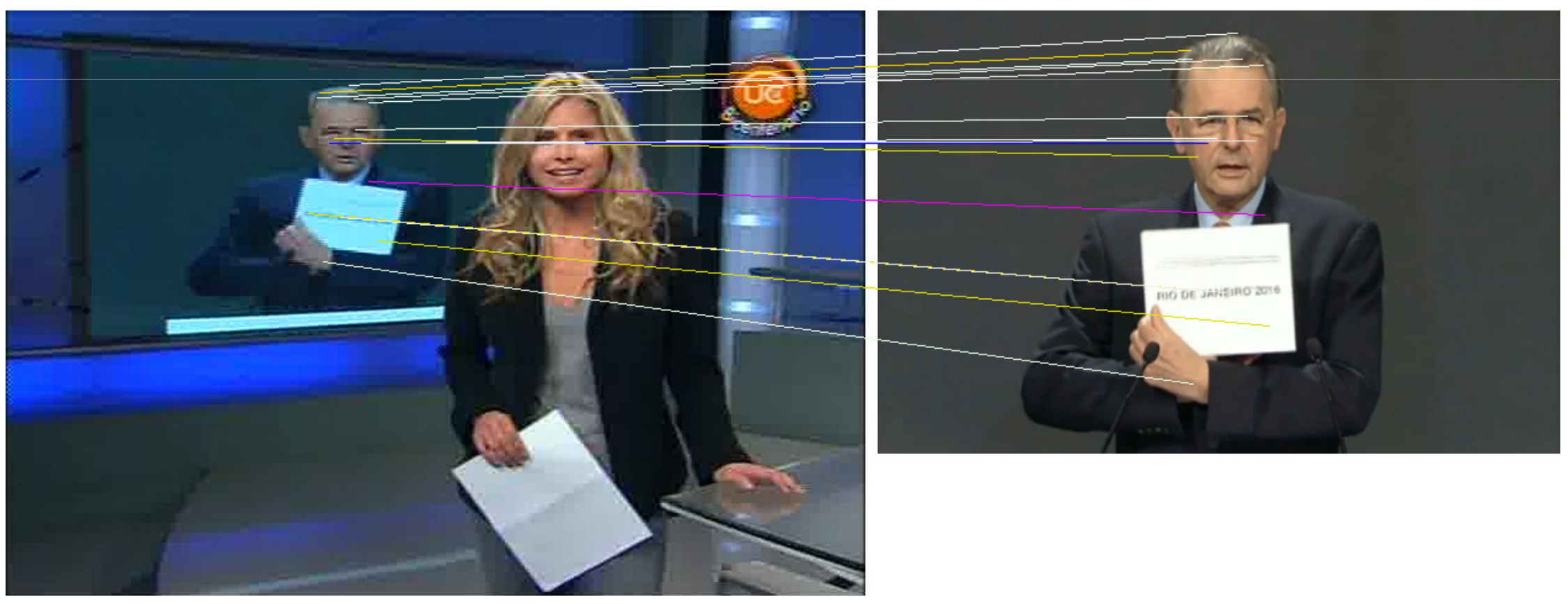
Case study 1 - image retrieval

- Big problem: semantic gap

- Bridge between high and low features
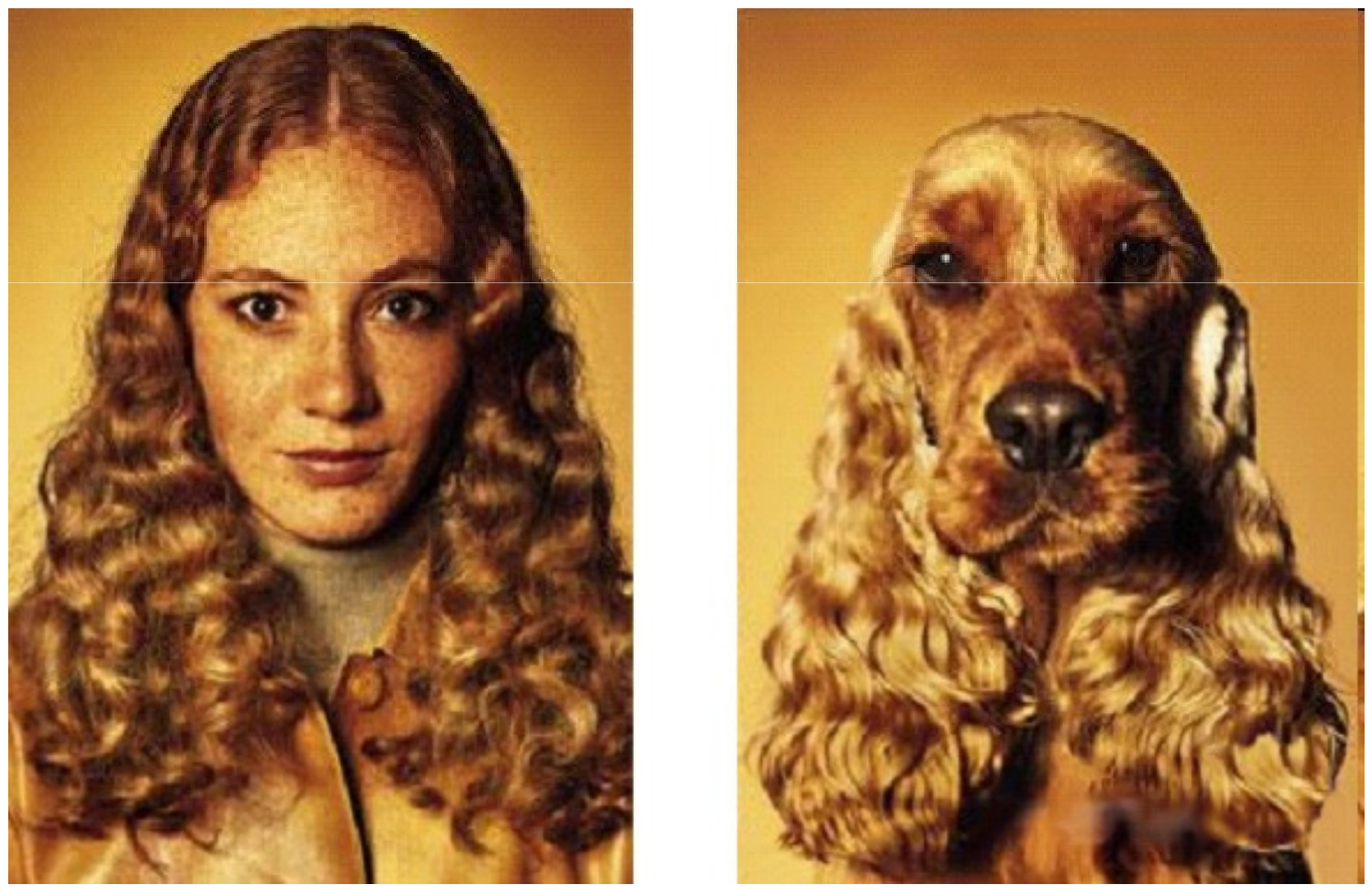

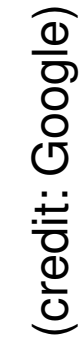




\section{Case study 1 - image retrieval}

- Non-metric functions for image retrieval

- $\chi^{2}$, Kullback-Leibler (KLD), Jeffrey divergence (JD)

$$
\begin{aligned}
& \delta_{\chi^{2}}(x, y)=\sum_{i=1}^{d} \frac{x_{i}-m(i)}{m(i)} \quad m(i)=\frac{x_{i}+y_{i}}{2} \\
& \delta_{K L D}(x, y)=\sum_{i=1}^{d} x_{i} \cdot \log \left(\frac{x_{i}}{y_{i}}\right) \\
& \delta_{J D}(x, y)=\sum_{i=1}^{d} x_{i} \cdot \log \left(\frac{x_{i}}{\frac{x_{i}+y_{i}}{2}}\right)+y_{i} \cdot \log \left(\frac{y_{i}}{\frac{x_{i}+y_{i}}{2}}\right)
\end{aligned}
$$

- Better suited for image retrieval and classification than metric distances 


\section{Case study 1 - image retrieval}

- Non-metric functions for image retrieval

- Dynamic Partial Function [Goh et al., 2002]

$$
\delta_{D P F}(x, y)=\left(\sum_{c_{i} \in \Delta_{m}}\left|x_{i}-y_{i}\right|^{p}\right)^{1 / p}, p \geq 1
$$

- $\Delta_{m}$ : set of $m$ smallest coordinate differences

- Better for image classification than Euclidean distance

- Fractional Lp distances

- Robust for image matching and retrieval

- Jeffrey divergence

- Better than Euclidean distance for retrieval of tomographies 


\section{Case study 2 - time series retrieval}

- The problem

- Time series $=$ ordered set of values

- Given a set of time series, find similar ones

- Find the optimal alignment

- $\mathrm{L}_{p}$ distance could be used, but:

- Scaling/different dimensionality

- Shift in time

- Missing values

- Outliers desired alignment

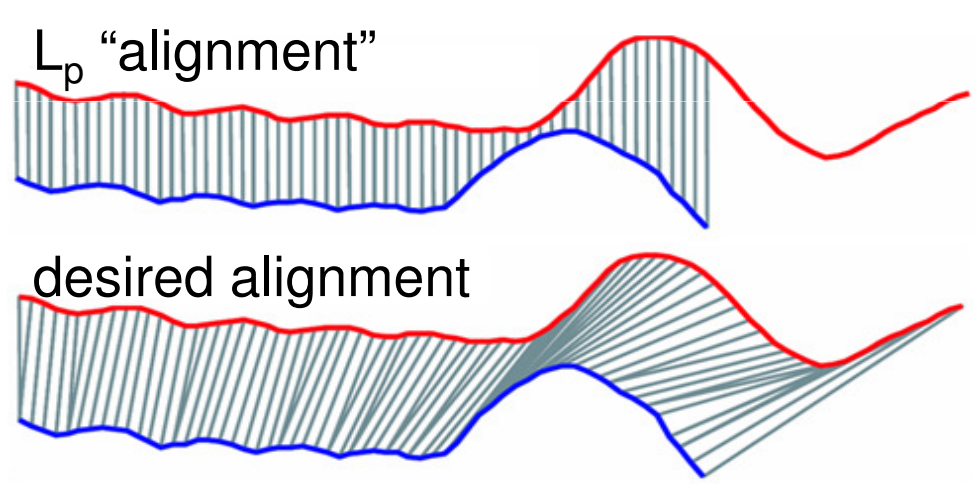

- Locality

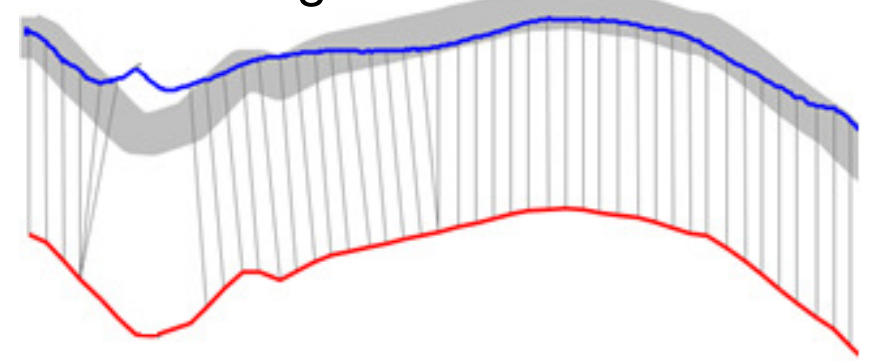




\section{Case study 2 - time series retrieval}

\section{- Applications}

- Financial analysis (e.g., stock prices)

- Medicine (e.g.,ECG, EEG)

- Scientific data (e.g., seismological analysis, climate data)

- Shape retrieval

- Many others...
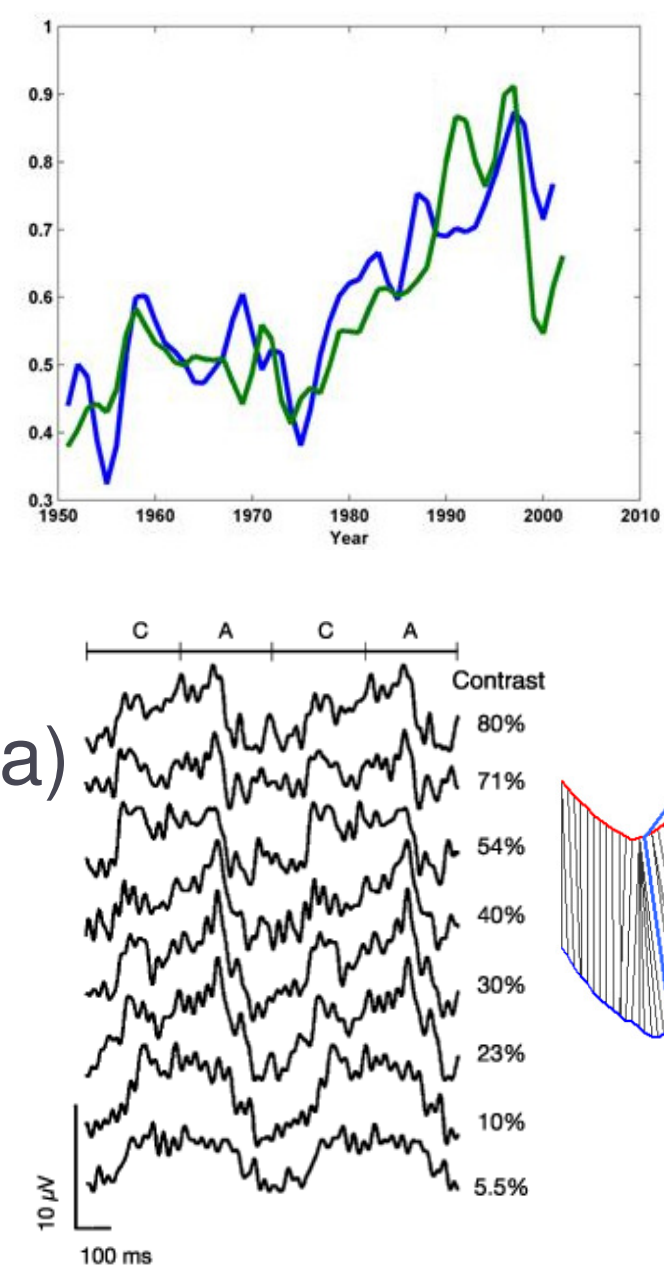


\section{Case study 2 - time series retrieval}

- Dynamic Time Warping (DTW) [Berndt and Clifford, 1994]

r. Sequences S1, S2

- $m \times n$ matrix $M$, where $m=\left|s_{1}\right|, n=\left|s_{2}\right|$

- Matrix cell $\mathrm{M}_{\mathrm{ij}}$ is partial distance $\mathrm{d}\left(\mathrm{s}_{\mathrm{i},}, \mathrm{S}_{2 \mathrm{j}}\right)$

$$
\delta_{D T W}(x, y)=\min _{W}\left\{\sqrt{\sum_{k=1}^{t} w_{k}}\right\}
$$

- Warping path $W=\left\{W_{1}, \ldots, W_{t}\right\}, \max \{m, n\}$ $\leq t \leq m+n-1$, is a set of cells from $M$ that are contiguous

1 $\mathrm{W}_{1}=\mathrm{M}_{1,1}, \mathrm{~W}_{\mathrm{t}}=\mathrm{M}_{\mathrm{m}, \mathrm{n}}$ (boundary condition)

D if $w_{k}=M_{a, b}$ and $w_{k-1}=M_{a^{\prime}, b^{\prime}}$, then

$\square \mathrm{a}-\mathrm{a}^{\prime} \leq 1 \mathrm{~b}-\mathrm{b}^{\prime} \leq 1$ (continuity)

$\square \mathrm{a}-\mathrm{a}^{\prime} \geq 0 \mathrm{~b}-\mathrm{b}^{\prime} \geq 0$ (monotonicity)

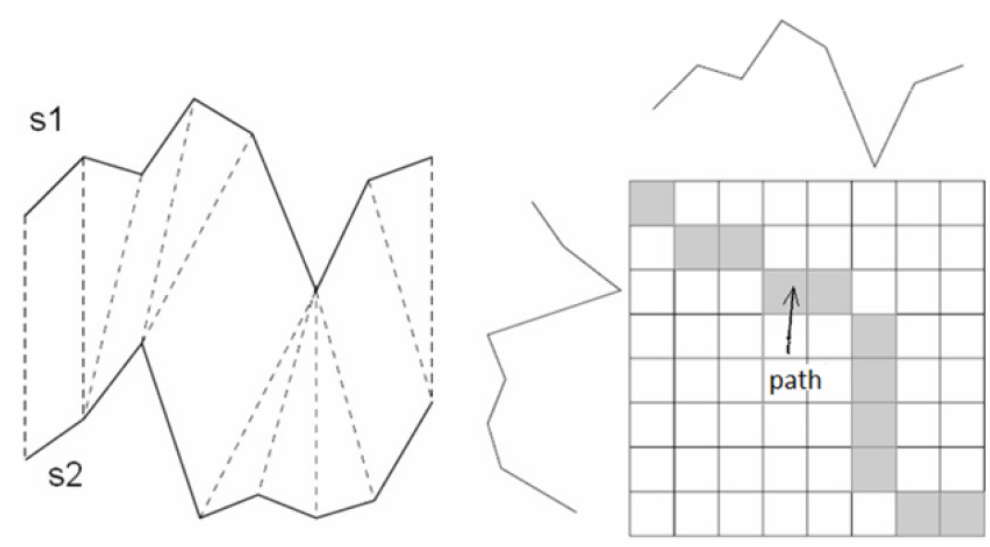

> $\mathrm{DTW}=\mathrm{L}_{2}$ distance on optimally aligned sequences (optimal warping path)

non-metric distance 


\section{Case study 2 - time series retrieval}

- Dynamic Time Warping (DTW)

- Exponentially many warping paths, but can be computed in $\mathrm{O}(\mathrm{mn})^{*} \mathrm{O}$ (ground distance) time by dynamic programming

v Constrained versions of DTW

- Avoiding pathological paths

$\square$ A range parameter $\omega$

$\square$ By $\omega=0, m=n, d(x, y)=|x-y|$ we get the Euclidean distance (just the diagonal warping path allowed)

- DTW reduced complexity to $\mathrm{O}((\mathrm{m}+\mathrm{n}) \omega)$

- Sakoe-Chiba band - warping paths are only allowed near the diagonal

" Itakura Parallelogram - "time warping" in the middle of sequences is allowed, but not at the ends

Sakoe-Chiba band

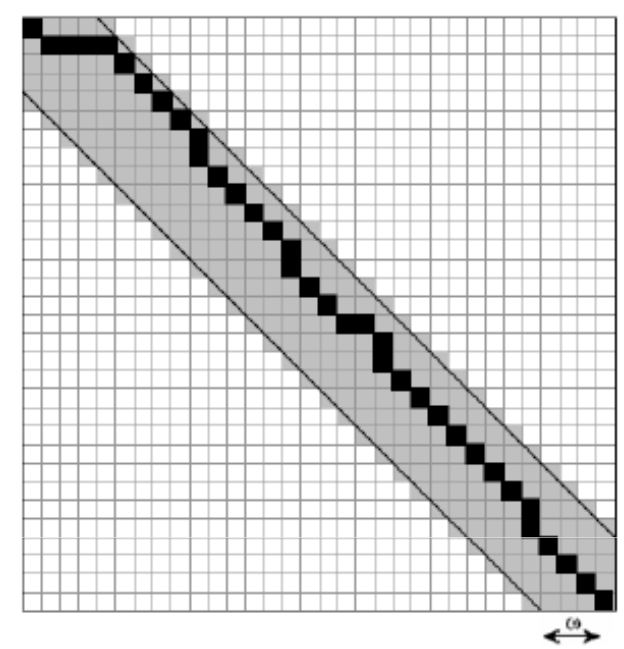

Itakura Parallelogram

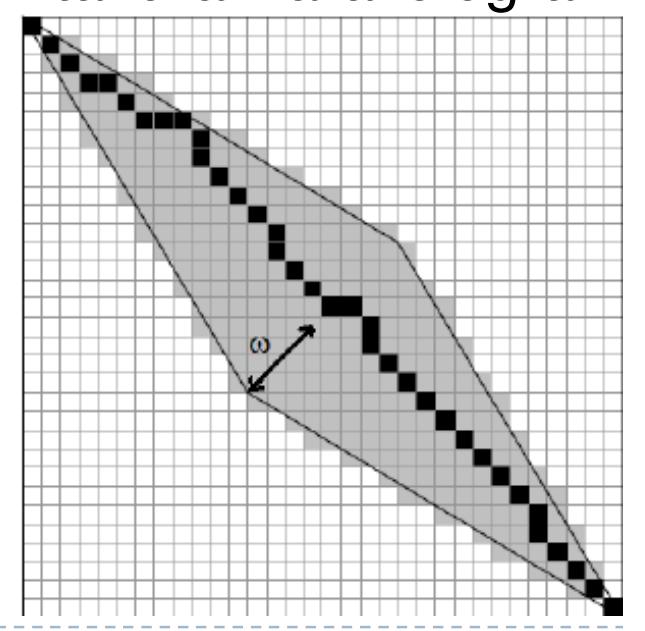




\section{Case study 2 - time series retrieval}

- Longest Common Subsequence (LCS)

r $x$ is subsequence of $y$ if there is a strictly increasing sequence of indices such that there is a match between symbols in $x$ and $y$ (not necessarily adjacent)

- $z$ is a common subsequence of $x$ and $y$ if it is a subsequence of both $x$ and $y$

- The longest common subsequence (LCS) is the maximum length common subsequence of $x$ and $y$

- non-metric (also similarity)

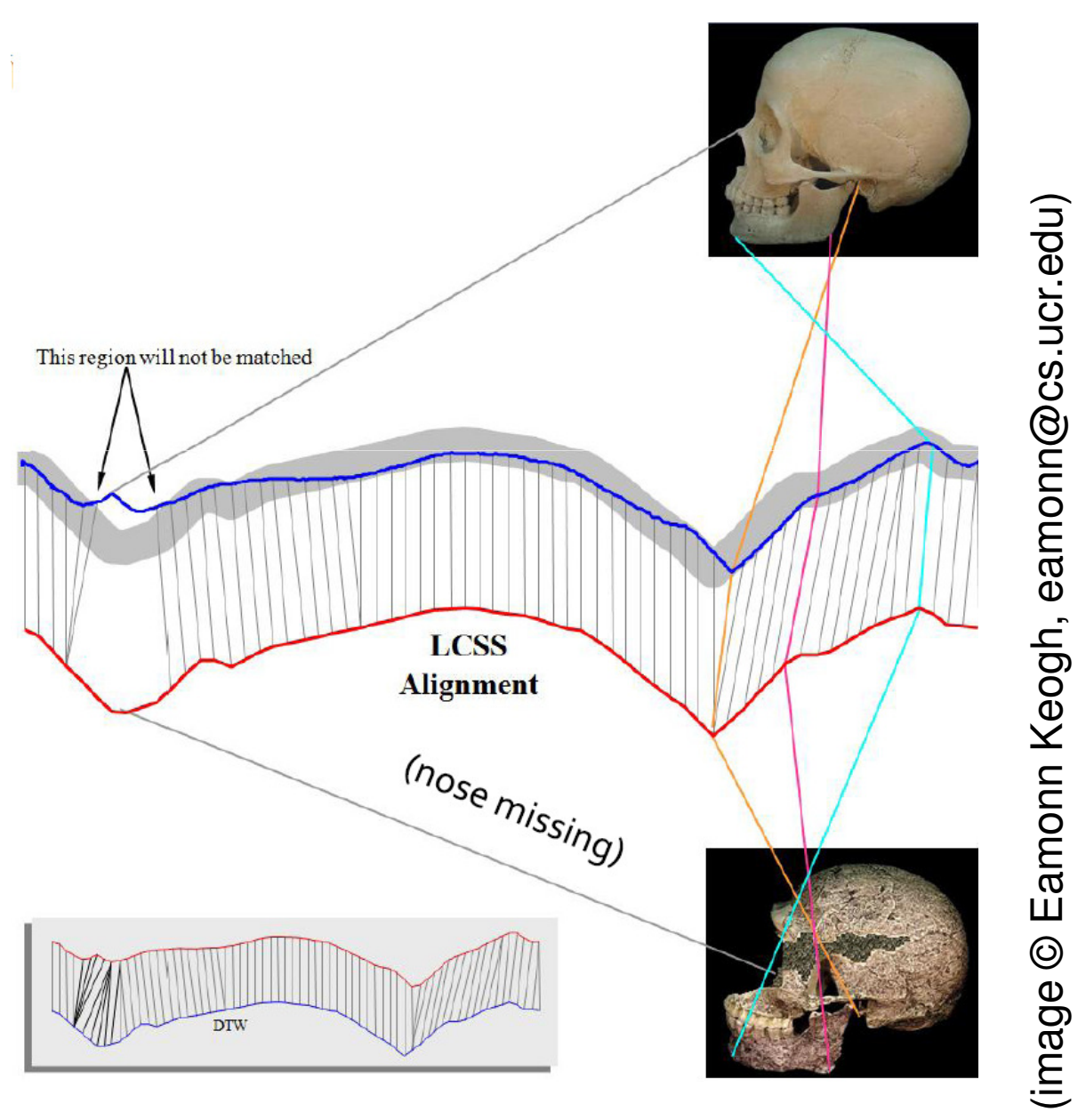


Case study 3 - protein retrieval

- Similar proteins $\rightarrow$ similar biological function

- Many applications, like protein function/structure prediction (leading to, e.g., drug discovery)

- Protein sequences (primary structure)

v Strings over 20-letter alphabet, i.e., symbolic chains of amino acids (AA)

- Biologically augmented string similarity

- Well-established model

- Protein structures (tertiary structure)

- 3D geometry (polyline + local chemical properties)

- Biologically augmented shape similarity

- Closer to function than sequence, harder to synthesize

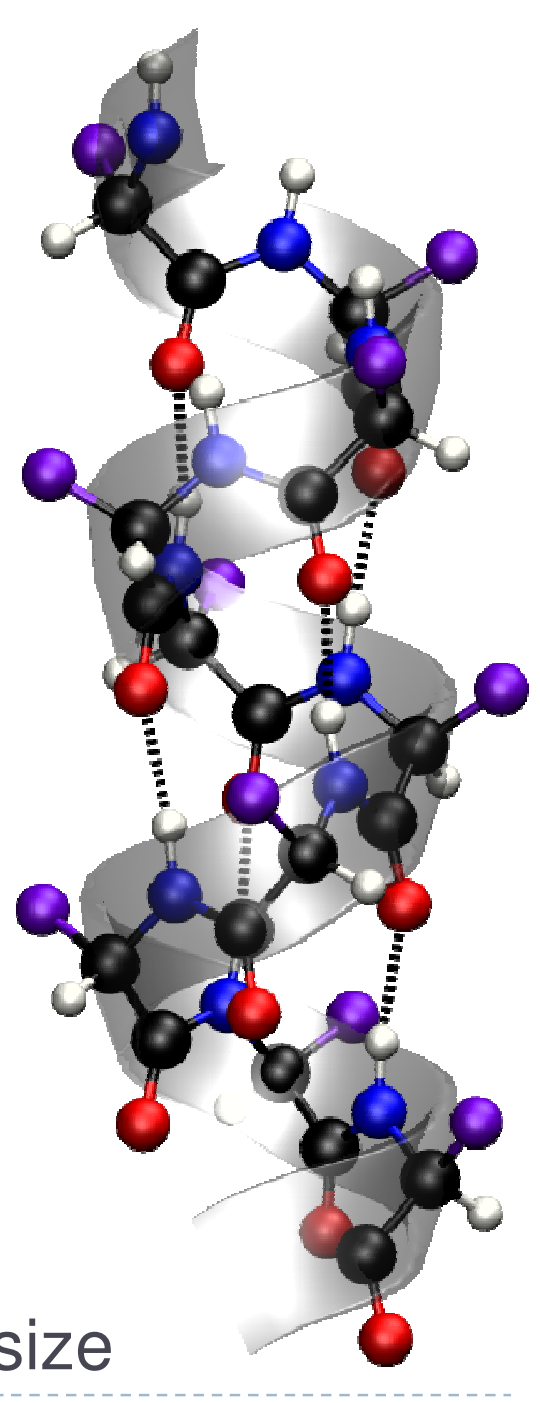
ICDE 2011, Hannover, Germany 


\section{Case study 3 - protein retrieval}

- Protein sequences

- String similarity (like edit distance) enhanced by scoring matrices (e.g., PAM, BLOSUM)

- Score between two letters models the probability of mutating one amino acid into the other

- Needleman-Wunch (NW)

- Global alignment - a nonmetric measure if scoring matrix is nonmetric and/or sequences are of different lengths

- Usually used for solving subtasks (e.g., when sequences are split into q-grams which are then indexed/searched)

- Smith-Waterman (SW)

- Local alignment (nonmetric), more applicable than global alignment

- BLAST - approximate SW + an access method in one algorithm

- Used for, e.g., function discovery, phylogenetic analysis, etc. 


\section{Case study 3 - protein retrieval}

\section{- Example}

- Global alignment (Needlemann-Wunch)

\begin{tabular}{ccccccccccccc|} 
N & P & H & G & I & I & M & G & L & A & E & & \\
-7 & -7 & +8 & +6 & -7 & -7 & +2 & +6 & +4 & -7 & -7 & $\rightarrow$ & -16 \\
- & - & H & G & - & - & L & G & L & - & - & & \\
\hline
\end{tabular}

- Local alignment (Smith-Waterman)

\begin{tabular}{|lllllllllllll|}
\hline N & P & H & G & I & I & M & G & L & A & E & & \\
& & +8 & +6 & +2 & & & & & & & $\rightarrow$ & 16 \\
& & H & G & L & & & & & & & & \\
\hline
\end{tabular}




\section{Case study 3 - protein retrieval}

- Protein structure

- Structure is more correlated to biological function than sequence (but harder to obtain)

- Similarity - two-step optimization process

1) Alignment of structures based on local properties/features

- Shape properties (torsion angles between AAs, density of AAs, curvature, surface area)

- Physico-chemical properties (hydrophobicity, AA volume)

2) Aggregation measure on top of the alignment

$\square$ RMSD, TM-score

- Existing top algorithms for function assessment

- DDPIn+iTM, PPM, Vorometric, TM-align, CE

[Hoksza \& Galgonek, 2010] 


\section{Case study 3 - protein retrieval}

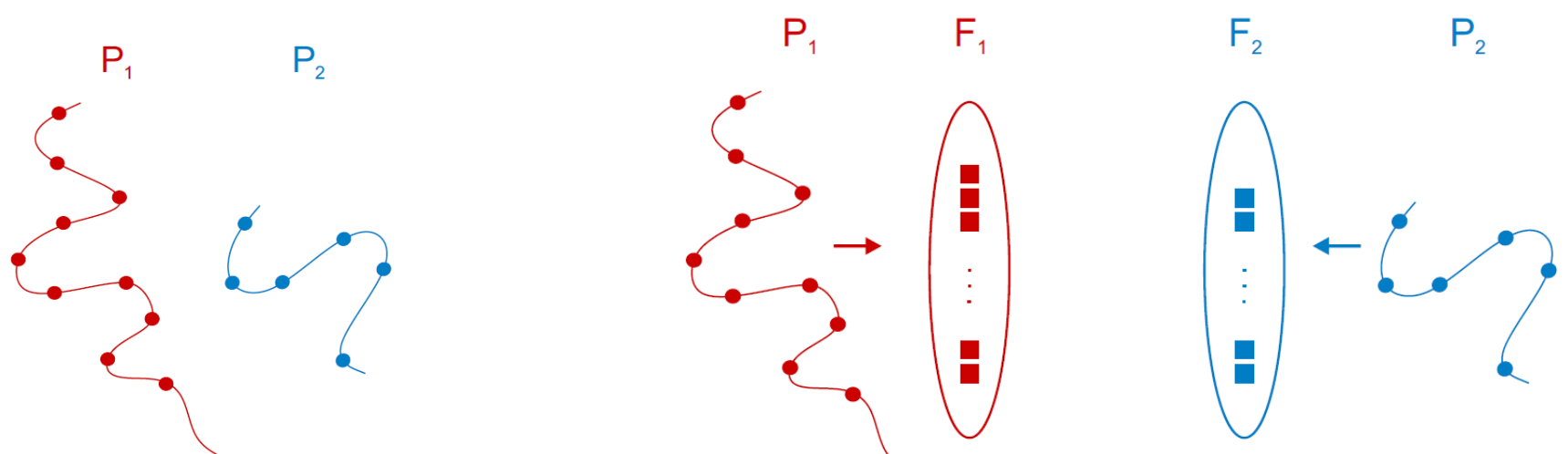

Proteins to compare
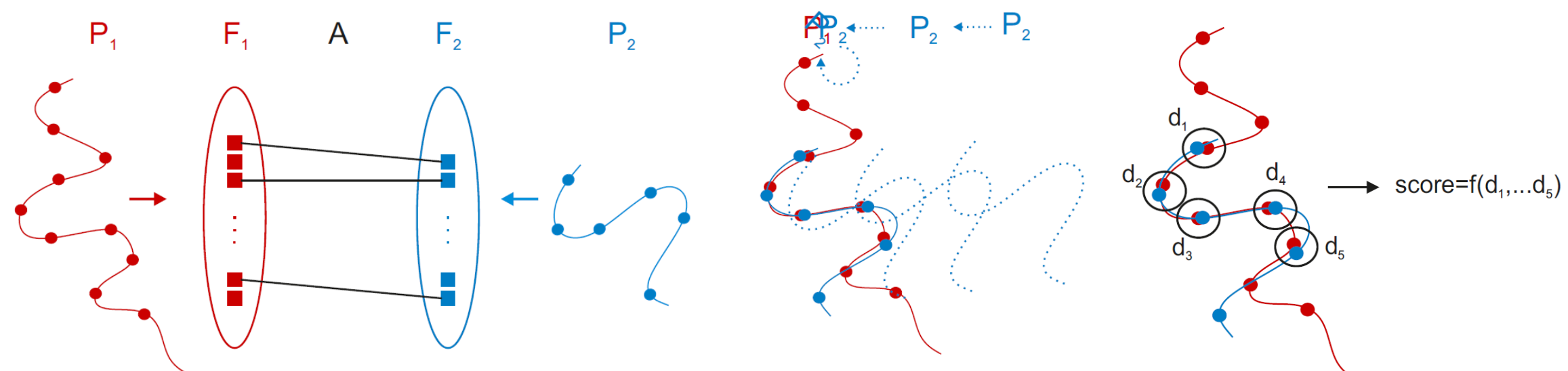

$\stackrel{\uparrow}{\longrightarrow}$ Local feature alignment 


\section{Indexing non-metric spaces - framework}

\section{- Need to search efficiently (fast query processing)}

- Access methods / indexes for similarity search

- Framework

- Metric case similarity

- MAM (metric access methods)

- Useful for mapping approaches

- General non-metric similarity

- General NAM (nonmetric AM)

- Black-box distance only

- Specific non-metric similarity

- Specific NAM

- Additional knowledge needed

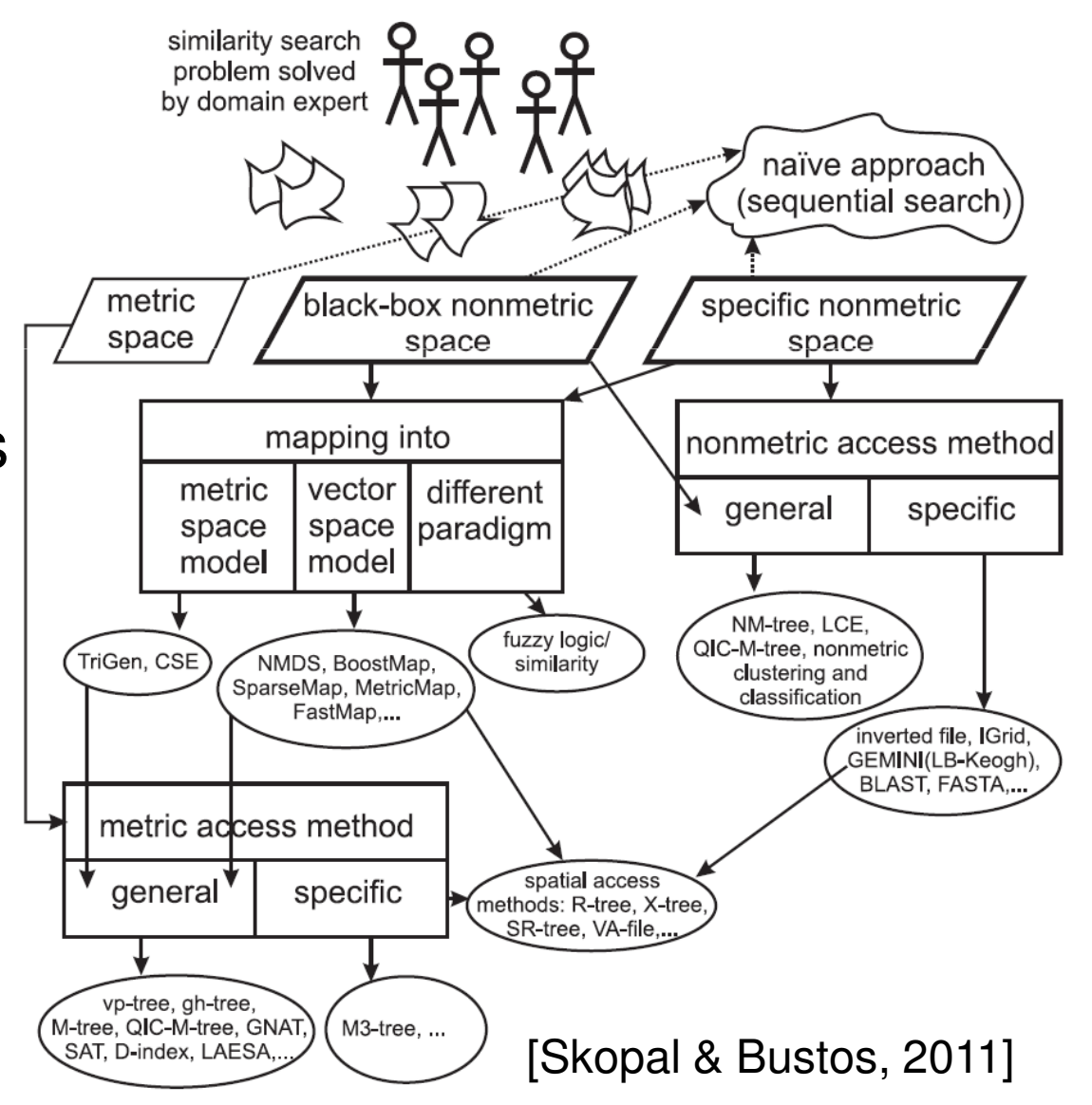




\section{Indexing non-metric spaces - MAM}

- The metric case (for completeness \& mapping approaches)

- Black-box metric distance $\delta$ needed

- Metric access methods (MAM), or metric indexes

r Idea: pivot-based lower-bounding

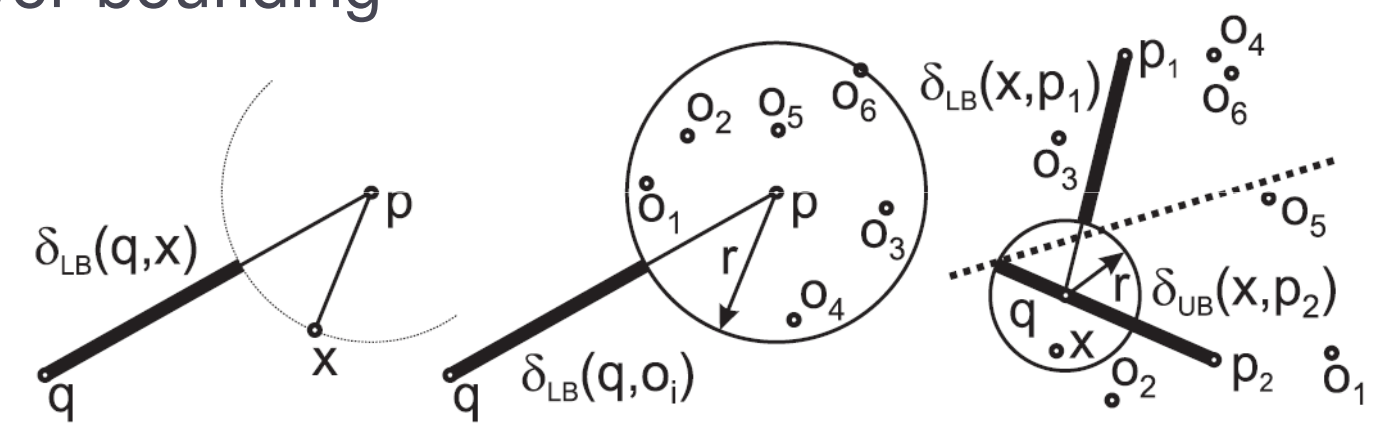

- Different implementations/designs [Zezula et al, 2005]

- Dynamic/static database, serial/parallel/distributed platform, main/secondary memory, exact/approximate search

- Index = set/hierarchy of metric regions, filtering

- Examples: M-tree family, pivot tables, vp-tree, GNAT, SAT, M-index, D-file, etc.

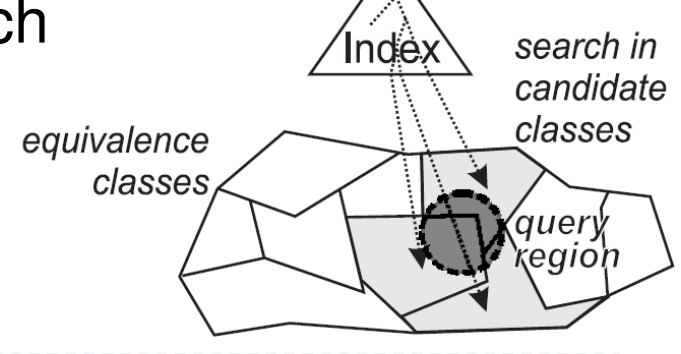




\section{Indexing non-metric spaces}

- MAM \& intrinsic dimensionality

- The metric postulates alone are not a guarantee of efficient indexing

- The structure of distance distribution indicates the indexability of the database

- Intrinsic dimensionality $\rho(\mathbf{S}, \boldsymbol{\delta})$ (idim) - an indexability indicator

[Chávez et al., 2001]

$$
\rho(\mathbb{S}, \delta)=\frac{\mu^{2}}{2 \sigma^{2}} \quad \begin{array}{r}
\left(\mu \text { and } \sigma^{2}\right. \text { are the mean and the variance of } \\
\text { the distance distribution in } \mathbf{S} \text { under } \delta)
\end{array}
$$
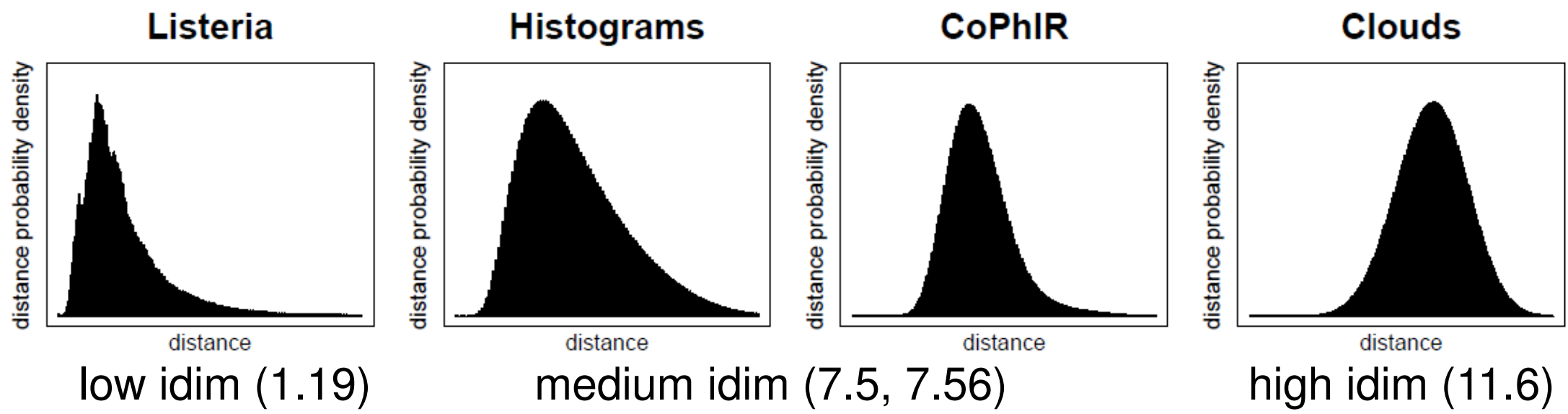


\section{Indexing non-metric spaces - mapping}

- How to index non-metric spaces?

- Let's simplify the problem, turn them into metric ones!

- Mapping into an $L_{p}$ space

1 Pros:

"Easy" target space (cheap $L_{p}$ distance, mostly Euclidean)

- Cons:

Approximate, static, computationally expensive mapping

- Variants of mappings into vector spaces

- Assuming metric distance

- FastMap, MetricMap, SparseMap, BoostMap

- Allowing also nonmetric distance

- Non-metric multidimensional scaling (NMDS) concept

- Query-sensitive embedding (non-metric extension of BoostMap) 


\section{Indexing non-metric spaces - mapping}

- Alternative mapping concept:

- Do not transform whole space (the database $\mathbf{S}+\delta$ ), but only the distance function $\delta$, leaving $\mathbf{S}$ unchanged

- Suppose semimetric distance $\delta$ (metric not satisfying triangle ineq.)

- How to turn semimetric $\delta$ into a metric?

- Consider increasing function $f$, such that $f(0)=0$, and modification $f(\delta)$ b i.e., f preserves the similarity ordering wrt any query

- Concave $f$ increases the amount of triangle inequality in $\delta$

- However, concave f also increases the intrinsic dimensionality of $(\mathbf{S}, f(\delta))$, when compared to $(\mathbf{S}, \delta)$

- Hence, let's find a function $f$ that is:

- Concave enough to turn $\delta$ into metric,

- yet keeping idim as low as possible

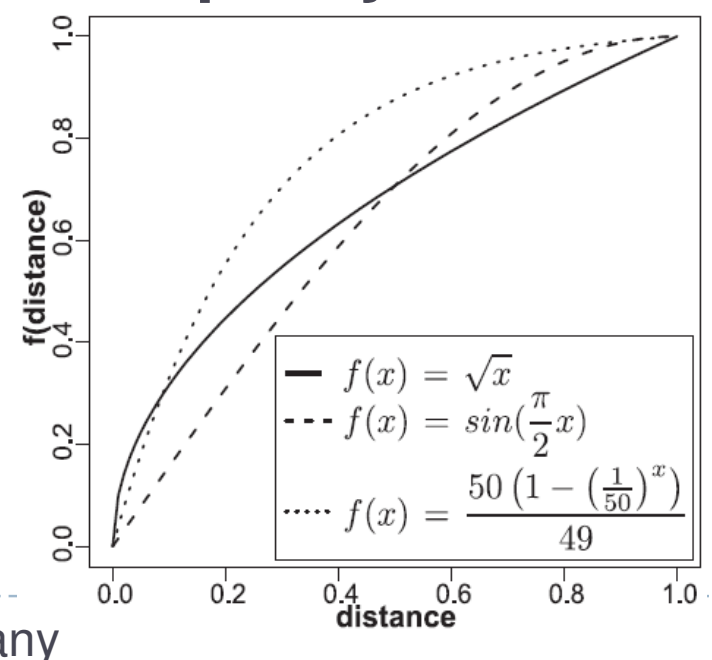




\section{Indexing non-metric spaces - mapping}

- TriGen algorithm [Skopal, 2007] Fractional-Power T-base

v "Metrization" of $\delta$ into $f(\delta)$

- Uses T-bases - set of modifying functions $f$, additionally parameterizable by a concavity/ convexity weight $\mathrm{w}$
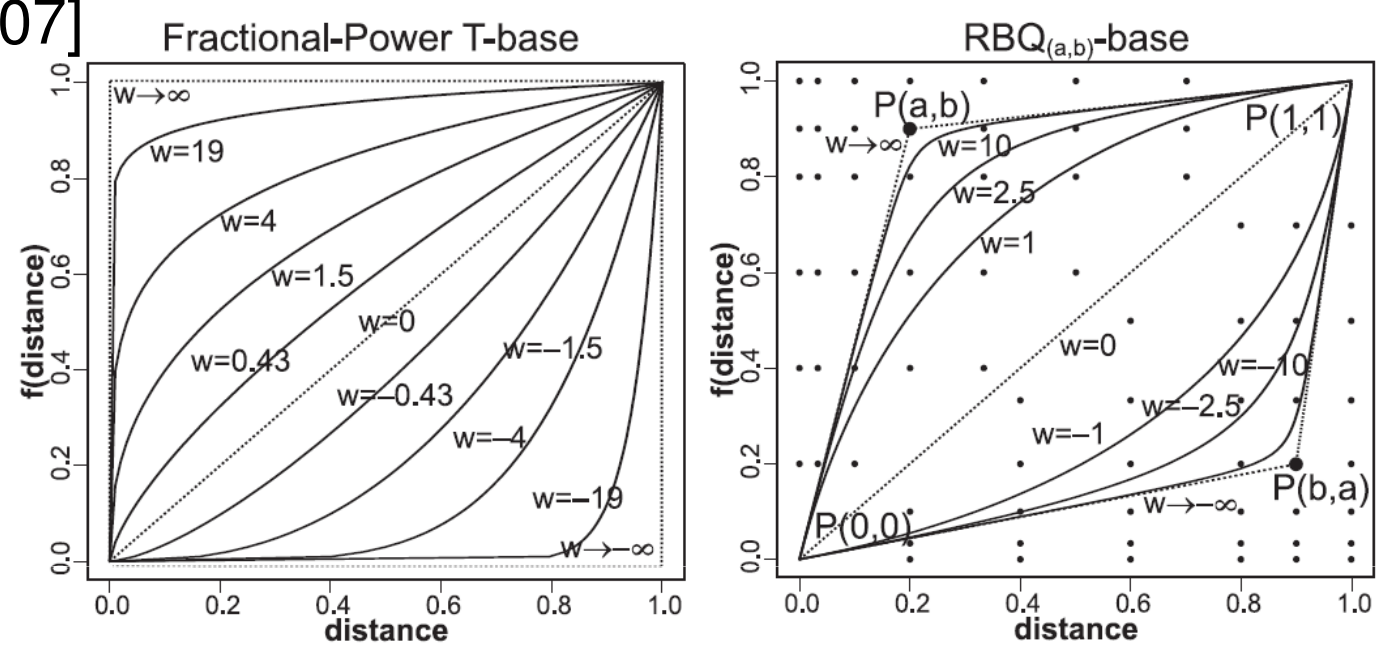

- Uses T-error - the proportion of non-triangle triplets

- Distance triplets sampled on $S$ using $f(\delta)$

- Given a set of T-bases, $\delta$ and a sample of the database $\mathbf{S}$, the algorithm finds the optimal $\mathbf{f}$ ( $\mathrm{T}$-base with $w)$

p $\mathrm{f}$ is a candidate if T-error is below a user-defined threshold $\theta$

- Among the candidates the one is chosen for which idim is minimal 


\section{Indexing non-metric spaces - general NAM}

- NM-tree - nonmetric M-tree

- M-tree combined with TriGen algorithm

- Allows to set the retrieval error vs. performance trade-off at query time

- The NM-tree idea [Skopal \& Lokoč, 2008]

- Using TriGen, find modifiers $\mathrm{f}_{\mathrm{i}}$ for several T-error thresholds (including zero T-error)

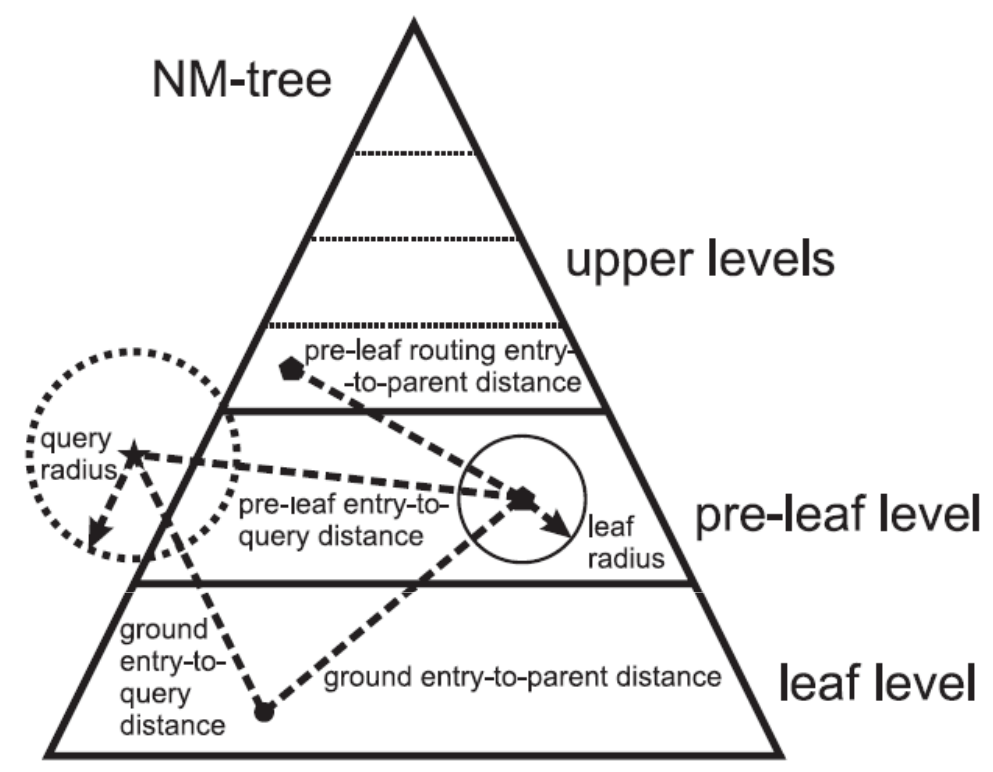

- Build M-tree using the zero T-error modified distance (i.e., full metric)

- At query time, the T-error tolerance is a parameter

- Each required distance value stored in M-tree is inversely modified from the metric one back to the original semimetric distance,

- then it is re-modified using a different modifier (appropriate to the query parameter)

- Additional requirement on T-bases - inverse symmetry, i.e., $f(f(x, w),-w)=x$ 


\section{Indexing non-metric spaces - specific NAM}

- The general techniques do not use any specific information

v just black-box distance and a sample of the database is provided

- It is always better to use a specific solution (if developed), based on an internal knowledge, as:

- Structure of the universe U (vector, string, set?)

- The formula of $\delta$ (closed form available?)

- Cardinality of the distance domain (discrete/continuous?)

- Data/distance distribution in $\mathbf{S}$ (uniform/skewed?)

- Typical query (e.g., sparse/dense vector?)

- Typically not reusable in other domains

- Hence, hard to find a NAM specific to our setup 


\section{Indexing non-metric spaces - specific NAM}

\section{- Example-LB_Keogh for constrained DTW}

[Keogh et al, 2006]

Lower-bounding distance, metric and cheap to compute $\mathrm{O}(\mathrm{n})$

- Envelope $W=\left(D T W \_U, D T W \_L\right)$ created for a time series $S$

DTW_ $U_{i}=\max \left(S_{i-R}: S_{i+R}\right)$,

DTW_L $L_{i}=\min \left(S_{i-R}: S_{i+R}\right)$,

$R$ is the thickness of Sakoe-Chiba band

$L B_{-} K \operatorname{Keog} h_{D T W}(Q, W)=\sqrt{\sum_{i=1}^{n}\left\{\begin{array}{cl}\left(q_{i}-D T W \_U_{i}\right)^{2} & \text { if } q_{i}>D T W \_U_{i} \\ \left(q_{i}-D T W_{-} L_{i}\right)^{2} & \text { if } q_{i}<D T W_{-} L_{i} \\ 0 & \text { otherwise }\end{array}\right.}$
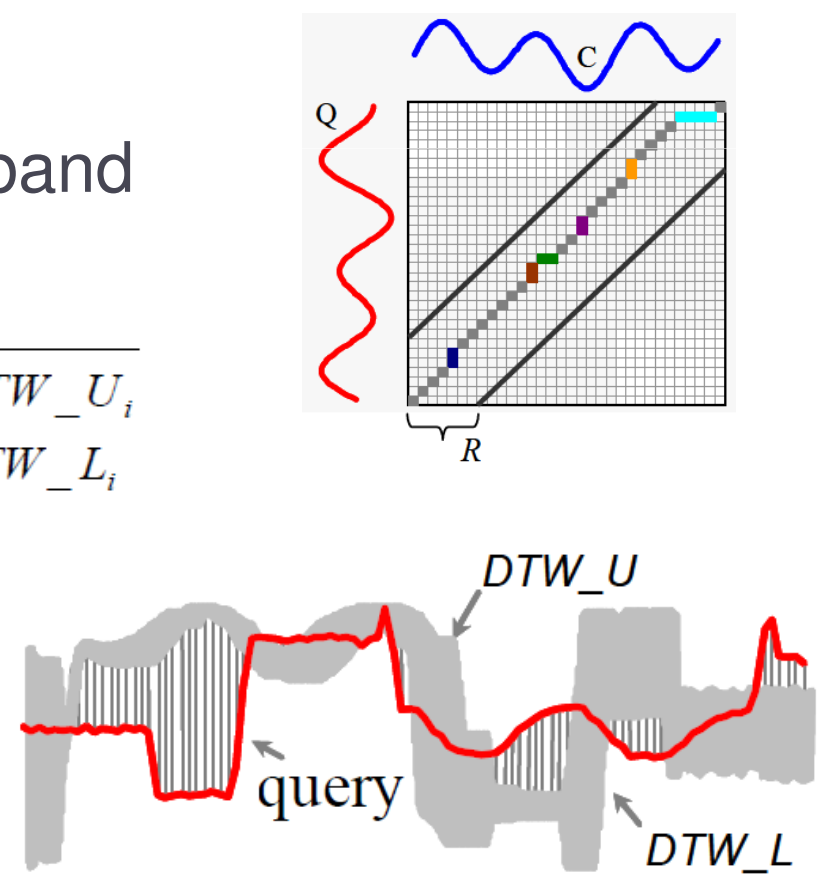

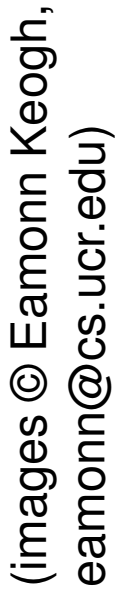




\section{Indexing non-metric spaces - specific NAM}

\section{- Example-LB_Keogh for constrained DTW}

- Basic approach - filter \& refine search

1) Sequential search under LB_Keogh

2) Check remaining candidates by DTW

- Extended approach - wedges

$=$ descriptors of multiple series

v Wedge $W=(U, L), U_{i}=\max \left(C_{1 i}, \ldots, C_{k i}\right), L_{i}=\min \left(C_{1 i}, \ldots, C_{k i}\right)$

- $\mathrm{W}=\mathrm{k}$-dimensional rectangle, let's index it by, e.g., R-tree

v For constrained DTW, W must be inflated as for single time series, i.e.,

DTW_U $\mathrm{U}_{\mathrm{i}}=\max \left(\mathrm{W}_{\mathrm{i}-\mathrm{R}}: \mathrm{W}_{\mathrm{i}+\mathrm{R}}\right)$,

DTW_L $L_{i}=\min \left(W_{i-R}: W_{i+R}\right)$

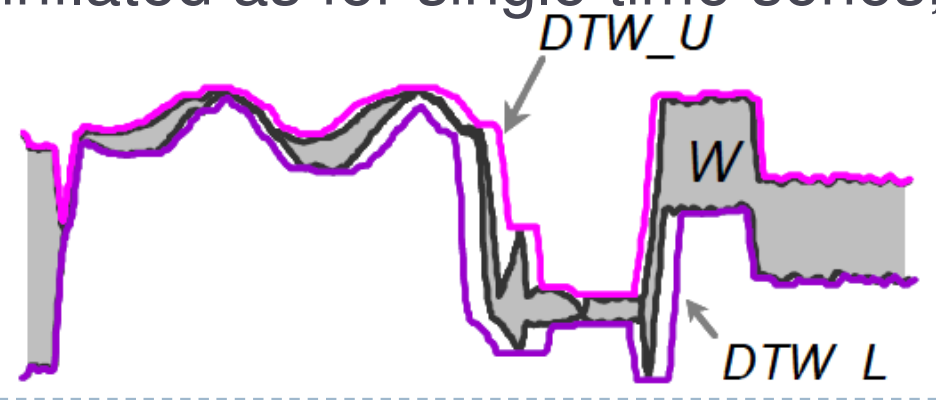




\section{Indexing non-metric spaces - specific NAM}

- Example - inverted file and cosine similarity

- Used as an implementation of range query in vector model of information retrieval

p documents $\mathbf{d}_{\mathrm{i}}$, terms $\mathrm{t}_{\mathrm{j}}$

- term-by-document matrix - weights of terms in documents

$$
\left(\begin{array}{lllll}
d_{1} & 0.6 & t_{2} & \ldots & 0.2 \\
d_{2} & 0 & 0 & \ldots & 0.1 \\
\vdots & : & : & & : \\
\vdots & : & : & & : \\
d_{n} & 0.2 & 0.5 & \ldots & 0.3
\end{array}\right)
$$

- Only efficient for cosine similarity (or inner product) and sparse query vector

- CosSim $=$ (normed) sum of weight multiplications

$$
\begin{aligned}
& \operatorname{Cos} \operatorname{Sim}\left(\boldsymbol{d}_{j} \boldsymbol{q}\right)= \\
& \frac{\vec{d}_{j} \cdot \vec{q}}{\left|\vec{d}_{j}\right| \cdot|\vec{q}|}=\frac{\sum_{i=1}^{t}\left(w_{i j} \cdot w_{i q}\right)}{\sqrt{\sum_{i=1}^{t} w_{i j}^{2} \cdot \sum_{i=1}^{t} w_{i q}^{2}}}
\end{aligned}
$$




\section{Indexing non-metric spaces - specific NAM}

- Example - inverted file and cosine similarity

- Efficient query processing

- Visit only lists of terms having nonzero weights in query

- Early termination provided when lists sorted wrt the weights

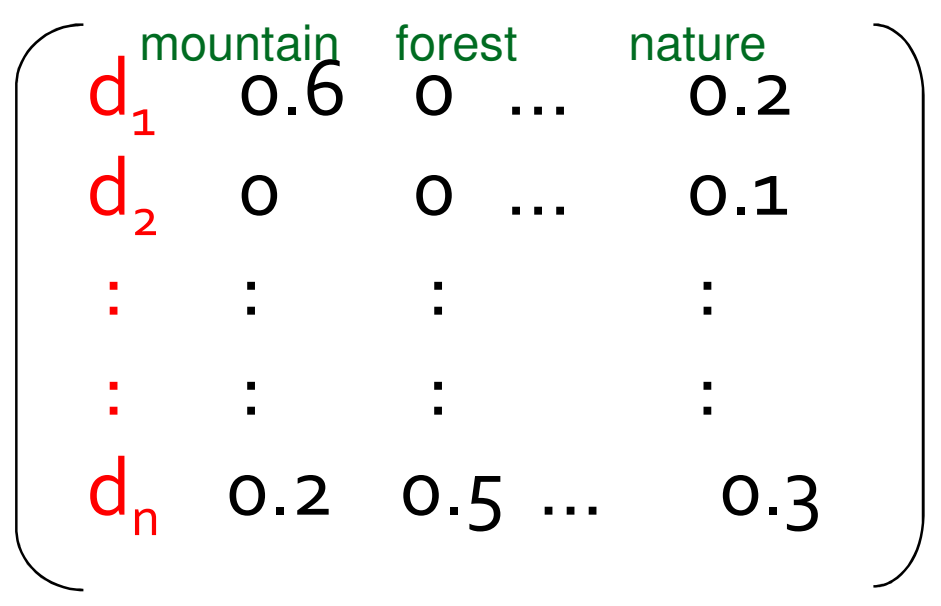

Query: $<0,0.5,0.4>$, similarity threshold $=0.05$, inner product used mountain $\rightarrow \mathrm{d}_{1}(0.6), \mathrm{d}_{\mathrm{n}}(0.2)$ forest $\rightarrow d_{n}(0.5)$

Answer:

... $d_{n}(0.37)$ nature $\rightarrow d_{n}(0.3), d_{1}(0.2), d_{2}(0.1) \quad d_{1}(0.08)$

- Cannot apply to Euclidean distance (!)

v zero + nonzero weight $=$ nonzero (all lists must be visited) 


\section{Indexing non-metric spaces}

- Overview of methods for efficient non-metric search

- References to the sections of [Skopal \& Bustos, 2011]

\begin{tabular}{|c|c|c|c|c|c|c|}
\hline Method & 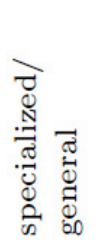 & 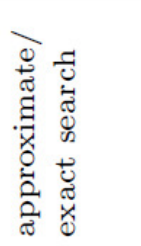 & 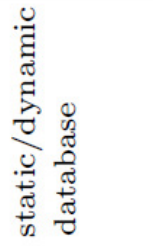 & 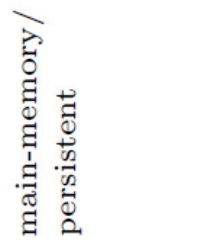 & 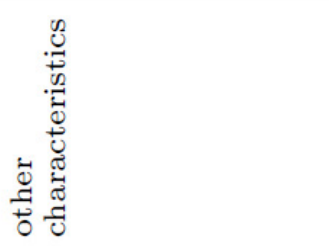 & 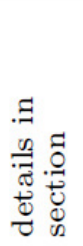 \\
\hline Sequential scan & Gen. & Exact & Dynamic & Both & Requires no index & $\mathrm{n} / \mathrm{a}$ \\
\hline CSE & Gen. & Exact & Static & Main-mem. & $\begin{array}{l}\text { Requires } \\
\text { space }\end{array}$ & 4.5 .2 \\
\hline TriGen & Gen. & Approx. & Static & Main-mem. & $\begin{array}{l}\text { Simplifies the prob- } \\
\text { lem to metric case }\end{array}$ & 4.5 .3 \\
\hline $\begin{array}{l}\text { Embeddings into } \\
\text { vector spaces }\end{array}$ & Gen. & Approx. & Static & Main-mem. & $\begin{array}{l}\text { Simplifies the prob- } \\
\text { lem to } L_{p} \text { space }\end{array}$ & 4.5 .4 \\
\hline Fuzzy logic & Gen. & Approx. & Static & Main-mem. & $\begin{array}{l}\text { Provides transitive } \\
\text { inequality, not im- } \\
\text { plemented yet }\end{array}$ & 4.5 .5 \\
\hline NM-tree & Gen. & Approx. & Dynamic & Persistent & $\begin{array}{l}\text { Based on M-tree, } \\
\text { uses TriGen }\end{array}$ & 4.6 .1 \\
\hline QIC-M-Tree & Gen. & Exact & Dynamic & Persistent & $\begin{array}{l}\text { Based on M-tree, } \\
\text { requires user-defined } \\
\text { metric lower bound } \\
\text { distance }\end{array}$ & 4.6 .2 \\
\hline $\mathrm{LCE}$ & Gen. & Approx. & Static & Main-mem. & $\begin{array}{l}\text { Exact only for } \\
\text { database objects }\end{array}$ & 4.6 .3 \\
\hline Classification & Gen. & Approx. & Static & Main-mem. & $\begin{array}{lr}\text { Requires } & \text { cluster } \\
\text { analysis, } & \text { limited } \\
\text { scalability } & \end{array}$ & 4.6 .4 \\
\hline $\begin{array}{l}\text { Combinatorial } \\
\text { approach }\end{array}$ & Gen. & Approx. & Static & Main-mem. & $\begin{array}{l}\text { No implementation } \\
\text { yet, only for NN } \\
\text { search. Exact for } \\
\text { large enough } D \text {. }\end{array}$ & 4.6 .5 \\
\hline Inverted file & Spec. & Exact & Dynamic & Persistent & Cosine measure & 4.7 .2 \\
\hline IGrid & Spec. & Exact & Static & Main-mem. & $\begin{array}{l}\text { Specific } L_{p} \text {-like dis- } \\
\text { tance }\end{array}$ & 4.7 .3 \\
\hline GEMINI(LB-Keogh) & Spec. & Exact & Both & Main-mem. & $\begin{array}{l}\text { Uses lower bound } \\
\text { distances }\end{array}$ & 4.7 .4 \\
\hline FASTA/BLAST & Spec. & Approx. & Dynamic & Main-mem. & $\begin{array}{l}\text { Approximate align- } \\
\text { ment }\end{array}$ & 4.7 .5 \\
\hline
\end{tabular}

ICDE 2011, Hannover, Germany 


\section{Challenges to the future}

\section{- scalability}

- mostly sequential scan nowadays, but the databases grow and get more complex, hence, indexing would be necessary

- indexability

- how to measure indexability of nonmetric spaces?

- implementation specificity

r specific vs. general NAMs

- efficiency vs. effectiveness

- slower exact vs. faster approximate search

- extensibility

v there exist other related aggregation/scoring (non-metric) concepts, to which non-metric indexing could contribute 


\section{Thank you for your attention!}

\section{... questions?}




\section{References}

- T. Skopal, B. Bustos, On Nonmetric Similarity Search Problems in Complex Domains, to appear in ACM Computing Surveys, 2011

http://siret.ms.mff.cuni.cz/skopal/pub/nmsurvey.pdf

- D. Berndt, J. Clifford. Using dynamic time warping to find patterns in time series. AAAI Workshop On Knowledge Discovery in Databases, 1994

- E. Chávez, G. Navarro, R. Baeza-Yates, J.L. Marroquín, Searching in metric spaces, ACM Computing Surveys, 33(3), 2001

- K.-S. Goh, B. Li, and E. Chang. DynDex: A dynamic and non-metric space indexer. 10th ACM International Conference on Multimedia, 2002

- D. Hoksza, J. Galgonek, Alignment-Based Extension to DDPIn Feature Extraction, International Journal of Computational Bioscience, Acta Press, 2010

- E. Keogh, L. Wei, X. Xi, S. Lee and M. Vlachos (2006) LB_Keogh Supports Exact Indexing of Shapes under Rotation Invariance with Arbitrary Representations and Distance Measures. VLDB 2006

- T. Skopal, Unified Framework for Fast Exact and Approximate Search in Dissimilarity Spaces, ACM Transactions on Database Systems, 32(4), 2007

- T. Skopal, J. Lokoč, NM-tree: Flexible Approximate Similarity Search in Metric and Non-metric Spaces, DEXA 2008, LNCS 5181, Springer

- P. Zezula, G. Amato, V. Dohnal, and M. Batko, Similarity Search: The Metric Space Approach, volume 32 of Advances in Database Systems. Springer, 2005 